\title{
Plume Tracking Strategy in Turbulent Environment Using Odor Sensor with Time Constant
}

\author{
Muis Muhtadi and Takamichi Nakamoto* \\ Tokyo Institute of Technology, 4259 Nagatsuta-cho, Midori-ku, Yokohama, Kanagawa 226-8503, Japan
}

(Received April 2, 2018; accepted June 22, 2018)

Keywords: source localization, plume tracking, turbulent airflow, computational fluid dynamics

The problem of tracking a dynamic odor plume using a realistic odor sensor model was investigated in a computer simulation. A chemical sensor typically has a significant time response delay that can disturb its practical application. This characteristic presents a challenge in tracking a dynamic plume toward the source. A reliable plume tracking strategy in a dynamic turbulent environment was designed through this simulation, which exploits the transient response of the chemical sensor. In this simulation, a mathematical model derived from a semiconductor gas sensor for sensing ethanol gas was used to model the sensor response. The sensor model was embedded into a chemotaxis-anemotaxis-based plume tracking strategy using a single sensor node to localize the source. In essence, the localization strategy is to search for the plume centerline and then move upwind along the centerline. The plume edges are estimated by evaluating the gradient of the fitting model of the frame size of the latest data points of sensor response during tracking. The strategy was tested on a turbulent environment simulated using a computational fluid dynamics (CFD) software. Although the odor distribution was complicated owing to the turbulent airflow and the obstacles, evaluation of the plume tracking strategy to localize the source starting from 404 initial positions achieved a success rate of approximately $70 \%$.

\section{Introduction}

Odor source localization has remained an interesting research topic in recent years owing to its potential contribution when such technology is successfully realized into application. This technology enables autonomous sensing systems to search for the origin of gas leakages under harmful or difficult situations and human victims in the event of disasters, for example.

There are several points to be solved in order to realize localization in a practical application. One of the issues is the fact that some commercially available chemical sensors typically have a significant response time delay especially in a real-time application task. ${ }^{(1)}$ Some works were carried out to model the response delay and considered it in the design of a source localization strategy. For example, Eu and Yap ${ }^{(2)}$ developed a sensing method to overcome the long recovery time of a metal-oxide gas sensor using evidential theory. Shigaki et al. ${ }^{(3)}$ developed

*Corresponding author: e-mail: nakamoto@nt.pi.titech.ac.jp https://dx.doi.org/10.18494/SAM.2018.1950 
a sensing method to estimate the input timing using an auto-regressive with exogeneous input (ARX) model. Ishida et al. ${ }^{(4)}$ proposed a control mechanism of a plume tracking robot on the basis of the transient response of a gas sensor. There has been an increasing interest in using the computational fluid dynamics (CFD) framework in the simulation of the odor source localization strategy. The CFD framework was used to build a realistic environment for evaluating source localization using a single robot or multiple robots. ${ }^{(5-9)}$

In this study, an odor plume tracking strategy using a gas and an anemometric sensor embedded in a mobile node is extended from the previous work ${ }^{(5)}$ by taking the gas sensor response delay into account. In this strategy, a new method of plume edge determination in a dynamic turbulent airflow based on the change in the transient sensor response over time is introduced. The strategy utilizes the transient sensor response, which corresponds to odor concentration, and flow direction to track the plume. The odor sensor has a response model derived from an actual semiconductor gas sensor. In this simulation experiment, a complicated circumstance is presented in which the search area is the floor of a closed room model with obstacles. Moreover, the tracking is started when an odor such as ethanol gas has been approximately distributed over the entire floor. The ethanol accumulation inside the room makes plume edge determination increasingly difficult with time.

The novelty of this work is the plume edge detection method, which uses a gradient sign computed from the sensor response function over time. This method detects the start edge of the plume by the negative gradient and the end of the plume by the positive one. There is no requirement of a threshold here, which potentially allows the application of this method for plume detection in any environment with any baseline concentration level.

Ultimately, this work contributes as follows. First, we built a testing environment using the CFD simulation to enable a thorough and repetitive evaluation of an odor source localization strategy under near-real-world circumstances. Second, the tracking strategy is designed to utilize a sensor with a response delay, demonstrating the feasibility of such sensor in practical application. Finally, the development of a localization strategy using a single sensor node equipped with an odor sensor and an anemometric sensor promotes further research for autonomous source localization using actual mobile robots.

\section{System Modeling}

\subsection{Simulation framework}

In this simulation experiment, the objective is to develop a two-dimensional (2D) odor plume tracking strategy for a turbulent environment, using an odor sensor with a time constant. The odor source is located on the floor. The turbulent environment is modeled using a CFD software, Fluent 17.2 (ANSYS, Inc.). The data of the modeled environment is then integrated into the plume tracking simulation in MATLAB (MathWorks, Inc.). 


\subsection{Odor distribution}

In the real-world environment, the odor source localization task generally encounters odor distribution in a turbulent environment. Assuming that odor is passive and neutrally buoyant, its distribution in such an environment is determined by the airflow dynamics. Among the various turbulent flow models available, we selected the standard $k-\varepsilon$ model based on Reynoldsaveraged Navier-Stokes (RANS) models. This model is defined by two transport equations that describe the behaviors of the kinetic energy $k$ and its dissipation rate $\varepsilon{ }^{(10)}$ In this model, it is assumed that the turbulent viscosity is isotropic in all directions. This turbulent model is established as simulation parameter in a CFD modeling of the environment.

\subsection{Testing environment}

A turbulent environment was simulated in the transient mode using the CFD software. It is a modification of the closed room model with one screen that we used in a previously reported work. ${ }^{(4)}$ It has now three screens $180 \mathrm{~cm}$ high and $50 \mathrm{~cm}$ wide as obstacles and the odor source is an outlet with a surface area of $25 \mathrm{~cm}^{2}$ emitting ethanol vapor continuously at $50 \mathrm{ml} / \mathrm{min}$ as shown in Fig. 1. This change presents higher complexity for source localization as more eddy areas are created downwind of the screens and the plume width is narrower than before. The physical parameters of the environment are shown in Table 1.

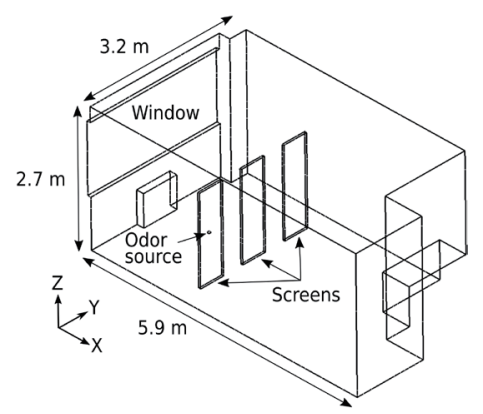

Fig. 1. Closed room model. The floor of the room is the searching area.

Table 1

Physical parameters.

\begin{tabular}{|c|c|}
\hline Parameter & Setting \\
\hline \multirow{2}{*}{ Odor source } & Ethanol gas $\left(\mathrm{C}_{2} \mathrm{H}_{5} \mathrm{OH}\right)$ \\
\hline & Emission rate: $50 \mathrm{ml} / \mathrm{min}$ \\
\hline \multirow{2}{*}{ Material } & Room boundaries: aluminum \\
\hline & Fluid: mixture of ethanol gas and air \\
\hline \multirow{2}{*}{ Temperature } & Window: $10^{\circ} \mathrm{C}$ \\
\hline & Others: $15^{\circ} \mathrm{C}$ \\
\hline Flow model & Standard $k-\varepsilon$ model \\
\hline Time step & $0.1 \mathrm{~s}$ \\
\hline Output & Coordinate, ethanol mass fraction, flow velocity \\
\hline
\end{tabular}


This modeling was executed in two phases: First, the simulation was conducted without odor release for $600 \mathrm{~s}$. During this time, natural convective airflow was gradually developed due to the difference between the temperature at the window $\left(10^{\circ} \mathrm{C}\right)$ and that at another part of the room $(15$ ${ }^{\circ} \mathrm{C}$ ). Second, it was continued with odor (ethanol) release from the gas outlet for $600 \mathrm{~s}$. These two phases resulted in time series of 3D data for 1200-s-long simulation consisting of odor concentration (ethanol mass fraction) and flow velocity irregularly distributed in space. Figure 2(a) shows airflow and ethanol distribution profile in ANSYS at $700 \mathrm{~s}$ and Fig. 2(b) shows the evolution of the ethanol mass fraction over time at point A in Fig. 2(a). The natural convective airflow in an enclosure is also known as internal natural convection. ${ }^{(11)}$ This airflow in general has laminar characteristics. However, the geometry and surface of the enclosure can disturb the flow and change its characteristics to become turbulent. ${ }^{(12)}$ As shown in Fig. 2, the obstacles disturb the flow and create turbulence at the downwind side of them.

In MATLAB, the data are processed with grid interpolation, resulting in uniformly distributed data. The interpolation grid size is $5 \mathrm{~cm}$. The source localization strategy searches for an odor source on the floor of the room model using 2D data throughout a horizontal plane 5 $\mathrm{cm}$ above the floor. Therefore, time-series $2 \mathrm{D}$ data at $5 \mathrm{~cm}$ above the floor was extracted from the 3D data. Moreover, only the data when odor had been released was used for the simulation.

\subsection{Sensor node model}

In this simulation, the sensor node (robot) is modeled as a mass point and it can move in any direction on the floor. Thus, it is assumed that the airflow is not disturbed by the sensor node's movement.

The sensor node measures odor concentration and flow direction, and checks for the existence of obstacles every $1 \mathrm{~s}$ interval while moving. During this process, necessary data are collected:

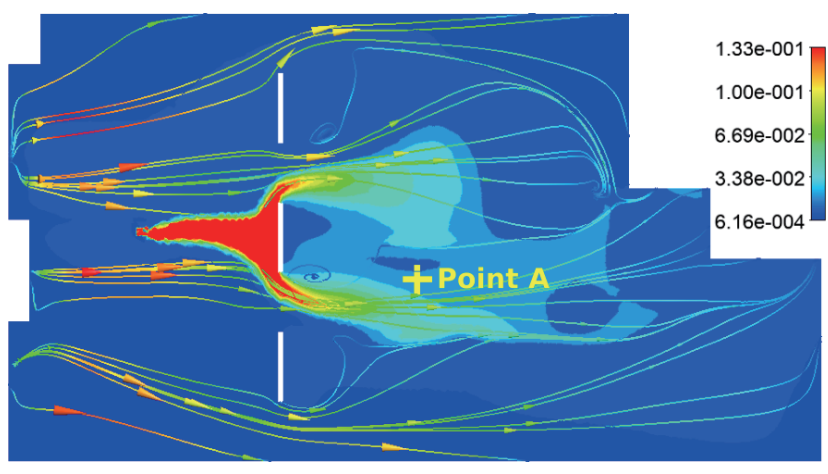

(a)

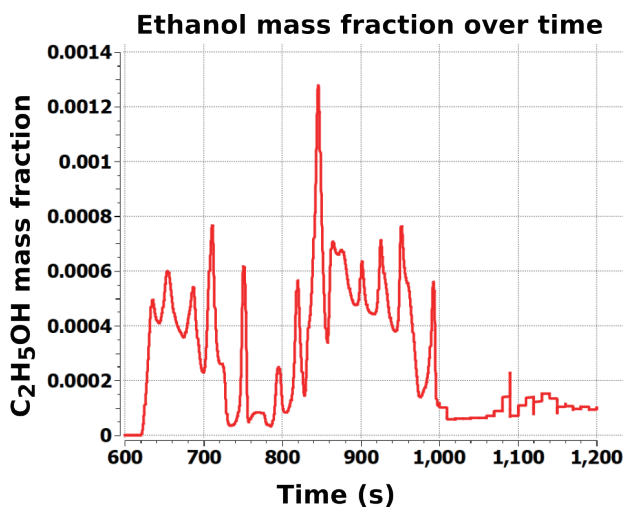

(b)

Fig. 2. (Color online) (a) 2D airflow profile and odor distribution at $5 \mathrm{~cm}$ above the floor at $700 \mathrm{~s}$ (ANSYS). (b) Ethanol mass fraction over time at point $\mathrm{A}$ in (a) at $5 \mathrm{~cm}$ above the floor. 
- observed sensor response $\left\{r\left(x_{i}, y_{i}, t_{i}\right)\right\}_{i=1}^{k}$ and

- flow velocity $\left\{u_{x}\left(x_{i}, y_{i}, t_{i}\right), u_{y}\left(x_{i}, y_{i}, t_{i}\right)\right\}_{i=1}^{k}$,

where $\left(x_{i}, y_{i}, t_{i}\right)$ is the $i$ th location and time when the data are collected, and $u_{x}$ and $u_{y}$ are the airflow velocities in the $x$ and $y$ directions, respectively. To perform the sensing task, the node is assumed to be equipped with an odor sensor, an anemometric sensor, an ultrasonic sensor, a GPS system, and a timer.

The odor sensor has a response model derived from an actual semiconductor gas sensor (TGS822, Figaro Engineering) for a remote-sensing source localization system. ${ }^{(13)}$ To simplify the model, the sensor response can be expressed as a function of time. The steady-state model of sensor response calibration over ethanol concentration is

$$
r_{0}(k)=\left[1+0.2309 C_{0}(k)\right]^{-0.6705}
$$

where $r_{0}(k)$ is the ideal sensor response (ratio of $R_{\text {gas }}$ to $R_{\text {air }}$ ) at time $k$ and $C_{0}$ is the ethanol concentration in ppm. From this equation, the maximum $r_{0}$ is equal to unity for zero concentration $C_{0}$. For sensor subsequent measurement at time $k$ and $k+1$, there is a time delay for the sensor response to change from $r_{0}$ to $r_{0}(k+1)$. If the delay is described by the first-order discrete-time system, the dynamic sensor response is obtained as

$$
r(k+1)=0.95 r(k)+0.05 r_{0}(k),
$$

where $r(k)$ and $r(k+1)$ are the observed sensor responses at time $k$ and $k+1$, respectively.

The sensing method by Eu and Yap ${ }^{(2)}$ requires some input parameters including the transient response and the threshold values for determining a certainty factor of odor detection. This method has complication in selecting the appropriate certainty factors and the threshold values, which should be different for different environmental conditions. This could discourage practical application. The auto-regressive model used in the sensing method by Shigaki et $a l .{ }^{(3)}$ is capable of modeling a wide range of nonlinear systems. However, the model function could contain an excessive number of terms and another method is required to determine the significant terms of this model. In order to comply with the real-time requirement, the challenge for using this method is to obtain a concise model of the sensor response.

In this study, the response dynamics is modeled on the basis of the sensor response characteristic to the target chemical and is independent of environmental conditions. Therefore, the model is computed only once for a particular sensor and is applicable for different kinds of environment. This simplicity allows the application of the method for any environment with different baseline concentration levels and increases the robustness against fluctuations.

An ultrasonic sensor is used to detect an obstacle so that the node can avoid it. It detects the obstacle within a $5 \mathrm{~cm}$ proximity in the direction $180^{\circ}$ ahead. In general, the obstacle is avoided by turning the tracking direction $90^{\circ}$ away from the direction to the obstacle. 


\section{Plume Tracking Strategy}

The main focus of our tracking strategy is source searching by tracking the plume. The tracking strategy here is an extension of the strategy that we reported previously. ${ }^{(5)}$ In previous work, the sensor was assumed to be ideal. It is capable of sensing any odor concentration and recovers from the response immediately. On the other hand, here, a realistic sensor model, whose sensor response shows delay, is used. Therefore, the new strategy is designed to exploit the transient sensor response and flow direction information to localize the source. The basic strategy is to look for the plume centerline and move upwind along the centerline toward the source.

\subsection{Tracking phase}

In this paper, a new simpler strategy than the previous one is proposed. As described in Fig. 3, the strategy has 3 phases.

- Upwind tracking: Tracking is started in phase. In this phase, the node moves in the upwind direction. It continues until it is declared that the node is out of the plume and then switches to 'crosswind scanning'.

- Crosswind scanning: Upon beginning 'crosswind scanning', the tracking node turns to the direction $90^{\circ}$ clockwise or counterclockwise from the upwind direction and then moves straight to search for the plume. The direction of the first turn, either clockwise or counterclockwise, is predetermined because there is no prior information of the plume location. However, the second turn onward is determined from the previously obtained plume location. In this phase, the search strategy is similar to the zigzag flying motion of a moth searching for a plume, i.e., the node moves in a crosswind direction for a certain distance to look for a plume edge. When the edge is not found within that distance, it moves in the opposite direction and searches for triple the previous distance and so on. This expanding zigzag search ends when a plume is found. Upon finding the plume, the plume centerline is determined from the minimum response found or the central point between the start and ending edges and then the phase switches to 'move to the centerline'.

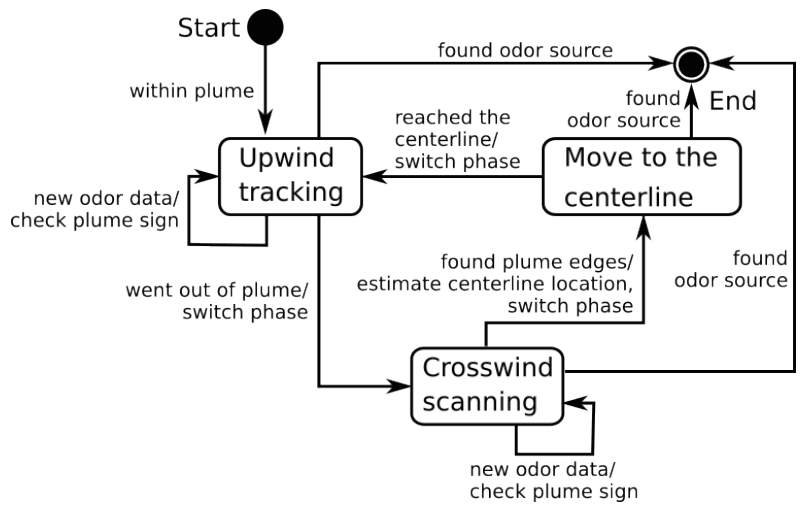

Fig. 3. Phases of plume tracking strategy. 
- Move to the centerline: In this phase, the node simply moves to the estimated centerline. Information on odor concentration and flow velocity is ignored during this phase. When the node has reached the estimated centerline, the phase switches to 'upwind tracking'. The determination of the plume edges and centerline is explained in Sect. 3.3.

\subsection{Tracking speed and obstacle avoidance}

As the sensor has a response delay, the faster the node moves, the less accurate the sensor response data it obtains. To compromise between the demand of fast tracking and appropriately accurate sensor response, we devised the sensor node (robot) with two speeds: high speed and low speed. The high speed is used during 'upwind tracking' and 'move to the centerline', whereas the low speed is used during 'crosswind scanning' as a more accurate response is considered necessary in this phase to estimate the centerline.

For selecting reasonable speeds, we took reference from a real experiment ${ }^{(4)}$ using a search robot with the same type of gas sensor as we used here. In that experiment, the robot moves at a speed of $10-20 \mathrm{~cm} / \mathrm{s}$ to localize an odor source in indoor space without an obstacle. In this study, more complication is provided by the turbulence created by the obstacles and the sensor response delay. Therefore, we selected the high speed to be $5 \mathrm{~cm} / \mathrm{s}$ and the low speed to be $2.5 \mathrm{~cm} / \mathrm{s}$. The speed values are indeed subject to optimization. However, the investigation for optimum speeds will be left as a future work.

The obstacle avoidance is prioritized over odor search. When detecting an obstacle, the robot stops searching/tracking for odor and turns $90^{\circ}$ and then moves, even if the odor information leads the robot toward the obstacle. This design is reasonable especially when it is known that the sensor response contains delay.

\subsection{Determination of plume edge and centerline}

A real-time method of detecting and tracking a dynamic plume is desirable. However, that is not possible owing to the sensor response delay. The plume is reliably indicated by the ideal sensor response $r_{0}$, but it is not accessible during plume tracking. However, the $r_{0}$ model can be derived through a calibration experiment. ${ }^{(13)}$ An investigation was performed in MATLAB to understand the correlation between the ideal response $r_{0}(k)$ and the observed response $r(k)$ by measuring the odor while moving the sensor node through a plume at a low speed. In this investigation, the real odor concentration is assumed to be available through the measurement and is used to calculate $r_{0}$. An example of the measurement track and the obtained responses are shown in Figs. 4(a) and 4(b), respectively. In Fig. 4(b), $r_{0}$ and $r$ are shown as the red and blue lines, respectively.

A transient sensor response function over time is obtained by applying curve fitting to the latest frame of $n$ data points of observed response $\left\{r\left(t_{i}\right)\right\}_{i=k-n+1}^{k}$ every time a new response value is obtained. The curve fitting is performed by the third-order polynomial regression technique. The fitting response function over time for time interval $\left[t_{k-n+1}, t_{k}\right]$ is 


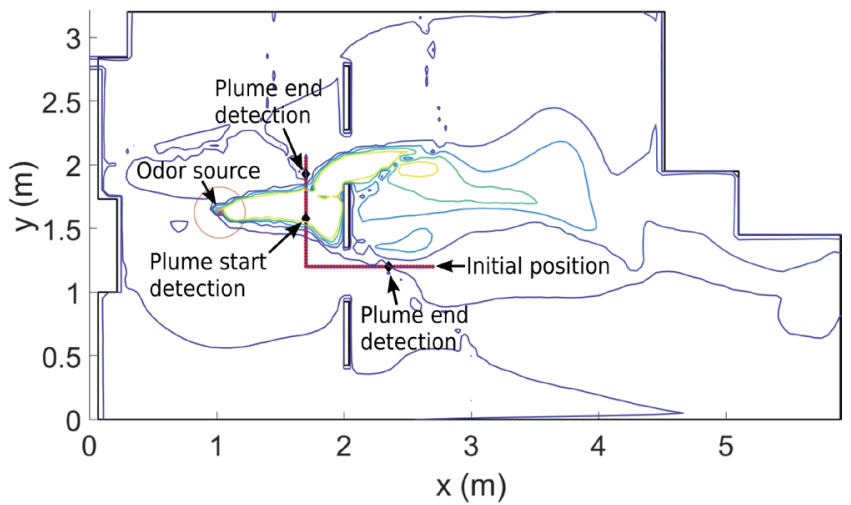

(a)

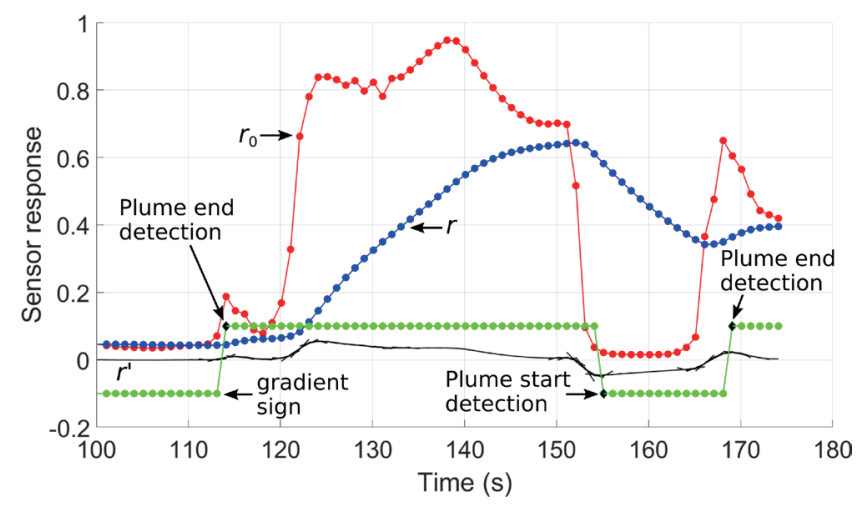

(b)

Fig. 4. (Color online) Odor measurement along an L-shaped track crossing a plume. (a) Measurement track. The contour lines indicate locations with the same concentration. (b) Sensor response during the measurement. The blue and red lines are the observed response $(r)$ and ideal response $\left(r_{0}\right)$, respectively. The black line is the successive plot of gradient function $r^{\prime}(t)$ for $\left[t_{(k-n+1)}, t_{k}\right]$. The green line is the sign of the gradient multiplied by 0.1 for observation purposes. The black markers in it indicate the detected start and end of the plume.

$$
\left\{\hat{r}(t)=w_{3} t^{3}+w_{2} t^{2}+w_{1} t+w_{0}\right\}_{t=t_{k-n+1}}^{t_{k}}
$$

where $\hat{r}(t)$ is the expected observed response at time $t$ and $w_{3}, w_{2}, w_{1}$, and $w_{0}$ are coefficients for minimizing the residual. The first derivative of $\hat{r}(t)$ is the gradient function of the observed response for time interval $\left[t_{k-n+1}, t_{k}\right]$, i.e.,

$$
\left\{r^{\prime}(t)=d \hat{r}(t) / d t\right\}_{t=t_{k-n+1}}^{t_{k}}
$$

This gradient describes the rate of response change over time. The successive plot of $r^{\prime}(t)$ for $\left[t_{k-n+1}, t_{k}\right]$ is shown as a black curve in Fig. 4(b). This curve shows that a positive $r^{\prime}$ correlates with an increase in $r_{0}$ and a negative $r^{\prime}$ correlates with a decrease in $r_{0}$. From Eq. (1), we know that a high $r_{0}$ corresponds to low concentration and a low $r_{0}$ corresponds to high concentration. As the gradient function is derived every time a new value is obtained at $t_{k}$, each data point obtained at time $t_{i} \geq t_{n}$ is included in the gradient calculation $n$ times in sequence, resulting in $n$ gradient values corresponding to each value. This repetitive calculation is handled by summing the overlapping gradient values for each data point. The sign of the summed gradient is shown as the green line in Fig. 4(b). The plume start and end are determined at the start and end of a gradient change to negative and positive, respectively, as marked by black markers in the green line.

The determination of the plume edge is a crucial problem in this strategy because it influences all the switching conditions of the phases. Since the gradient is calculated using $n$ data points, at the beginning of tracking, the first calculation is started when the obtained data 
is equal to $n$ data points. This causes a delay in judging the plume edge by $(n-1)$ data points. Apart from the sensor response delay, another issue in determining the edges is that turbulent airflow can create a pocket of highly concentrated odor separated from the main plume. Odor measurement through this pocket can present a gradient sign change that leads to the incorrect determination of the plume edge. This issue can be solved by the redundant checking of the gradient sign before declaring the plume edge. The declaration of edge detection is made when the gradient sign is consistent every time new sensor data is obtained for several detections. This method adds more delay in edge detection but can improve its accuracy.

The plume centerline is searched during 'crosswind scanning'. We investigate two methods of estimating the centerline. The first is a search for the minimum sensor response that corresponds to the maximum odor concentration within the plume. The second is to calculate the central point between the start and end of the plume.

In this simulation, it is assumed that a GPS system provides an absolute position. The position information is used to pinpoint the estimated centerline location in the end of crosswind scanning (see Fig. 3). Although the exact requirement for accuracy was not investigated, the acceptable error for the centerline coordinate in the crosswind direction should be smaller than half of the crosswind width of the plume so that the estimated location is still inside the plume.

\section{Plume Tracking Simulation}

\subsection{Methodology}

The environment changes every $0.1 \mathrm{~s}$ as defined in the ANSYS parameters. The tracking is started when odor has been continuously released for $100 \mathrm{~s}$, by which time, a relatively steady odor plume has developed. The time duration to localize the source is $500 \mathrm{~s}$. The tracking node is assumed to have found the odor source successfully if it arrives within $20 \mathrm{~cm}$ from the source location before the time limit. The frame size $n$ for the curve fitting of sensor responses is 5 data points. The redundant check of the gradient sign change is performed 5 times. In order to increase the robustness of upwind tracking against eddies and turbulent flow, the upwind direction during 'upwind tracking' is determined by averaging the flow direction of the last 5 data points.

In this simulation, the performances of tracking were compared between the two methods of centerline determination. The overall performance is evaluated by running the tracking simulation starting from 404 initial positions. The performance parameters are the average time required to localize the source, the standard deviation, and success rate, i.e., the ratio of the number of tracking cases in which the odor source was successfully found before the time limit to the total number of tracking cases. 


\subsection{Result and discussion}

A case of tracking simulation starting from the initial position $(2.2,1.2)$ is shown in Fig. 5(a) and the sensor response is shown in Fig. 5(b). In Fig. 5(a), the directions and sequence of the tracking phases are indicated by black arrows with numbers. The tracking starts with 'upwind tracking' (1), then 'crosswind scanning' (2), then 'move to the centerline' (3), and finally 'upwind tracking' (4) until the node arrives within $20 \mathrm{~cm}$ of the source location (indicated by the red circle around the source location).

In Fig. 5(b), the red line is the ideal sensor response $\left(r_{0}\right)$, the blue line is the observed sensor response $(r)$, the black line is the framewise gradient of sensor response $\left(r^{\prime}\right)$, and the green line is the gradient sign multiplied by 0.1 to aid in easy observation. From the green line, the state of the node when the corresponding sensor response is obtained either inside or outside a plume can be observed. The node is assumed to be inside the plume when the line is negative and outside when it is positive. The marker shape on the green line indicates the phase state, i.e., $\bullet$ for 'upwind tracking', - for 'crosswind scanning', and * for 'move to the centerline'. As shown in the green line, the tracking phase is not switched at the first plume edge detection, rather after 9 data points owing to the 4-data-points delay in judging the edge $(n-1)$ and the 5-datapoints delay for the redundant check.

The overall tracking performances are evaluated and compared for the two centerline determination methods, i.e., phase based on (a) minimum response and (b) central point between the start and ending edges, as shown in Fig. 6. In the figures, the time performance of tracking starting from an initial position is represented by colored cells at the coordinates of the initial position. In this context, failure, that is, the odor source has not yet been localized by the maximum allowed time, is represented by yellow. The figure shows that the successful cases, regardless of the centerline determination method, have initial positions with high odor concentration or near the plume centerline. However, the determination method using the central point showed better performance for initial positions far from the source at

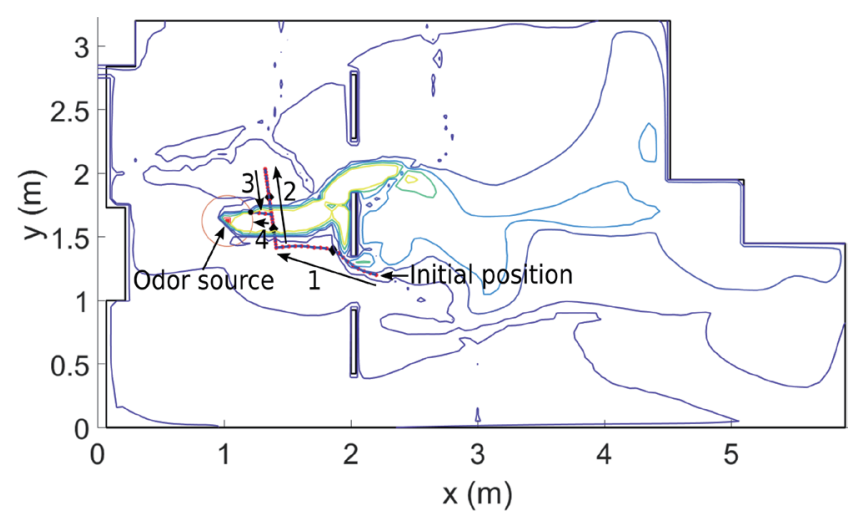

(a)

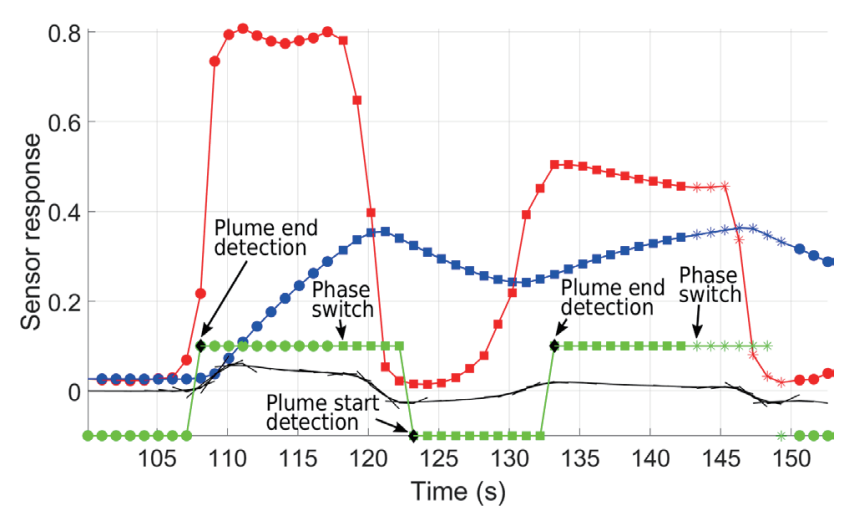

(b)

Fig. 5. (Color online) (a) Plume tracking starting from initial position $(2.2,1.2)$. The contour lines indicate locations with the same concentration. (b) Sensor response recorded during tracking. 


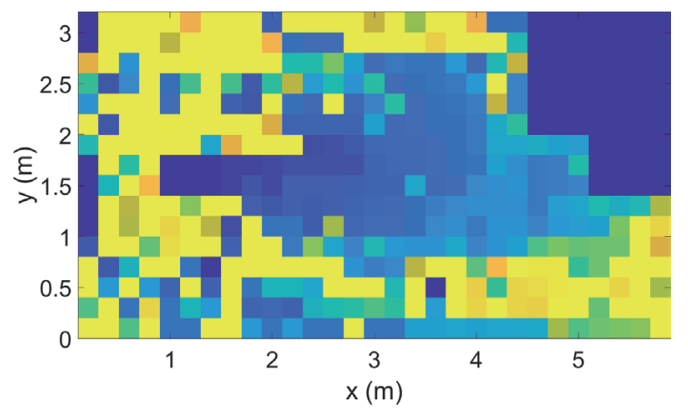

(a)

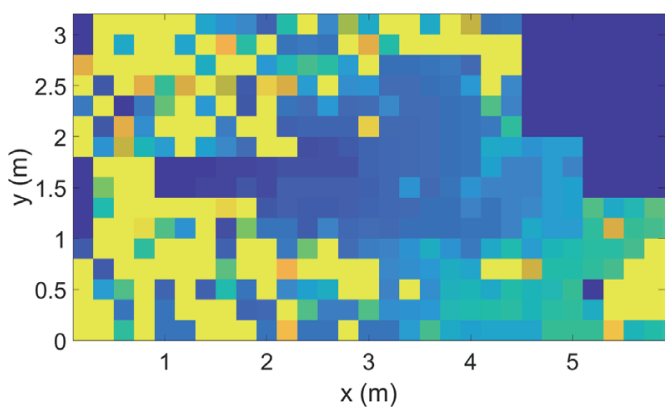

(b)

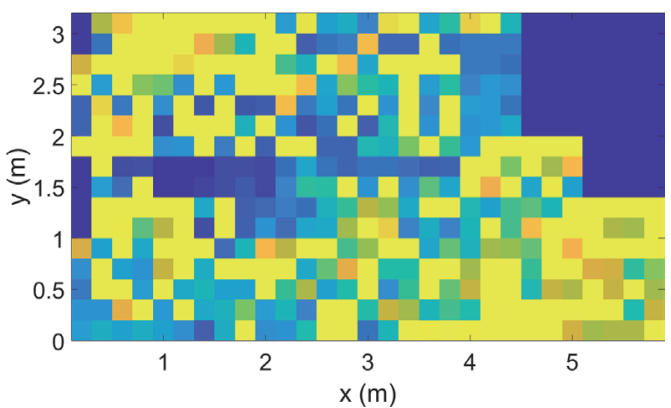

(c)

Fig. 6. (Color online) Time performance distribution depending on initial position. Tracking strategy with response delay consideration in which the centerline is estimated during 'crosswind scanning' on the basis of (a) minimum response and (b) central point between start and ending edges. (c) Tracking strategy without response delay consideration in which the centerline is estimated during 'crosswind tracking' based on minimum response.

the downwind side. The time performance with initial positions far from the centerline is generally long, typically owing to failure in finding the plume. This failure might correlate with the design selection of the strategy in which tracking starts with 'upwind tracking'. The performance when initial positions are at the upwind side of the source is also generally long as this tracking strategy is basically designed only to move crosswind to search for the plume centerline and move upwind along the centerline and does not have a suitable method of searching for the plume in the downwind direction.

The performance statistics of the strategy with and without time delay consideration are shown in Table 2. The tracking strategies with response delay consideration perform better as they show significantly higher success rates and lower average times to localize the source than does strategy without delay consideration. ${ }^{(4)}$ Among the strategies considering response delay, the one using centerline determination based on a central point is statistically better. This finding suggests that centerline determination based on the central point is more accurate. Centerline determination using the minimum response is less accurate owing to the sensor response delay. Further investigation indicates that the inaccurate determination of the centerline can lead to missing the plume completely for the narrow plume. 
Table 2

Performance of tracking for different centerline determination method. The search was carried out from 404 initial positions and the time limit for each duration is $500 \mathrm{~s}$.

\begin{tabular}{lcccc}
\hline \multirow{2}{*}{ Tracking strategy } & Centerline determination by & \multicolumn{2}{c}{ Time performance (s) } & Success rate \\
\cline { 2 - 4 } With response delay consideration & Mean & Std. deviation & (\%) \\
\hline & $\begin{array}{c}\text { Minimum response } \\
\text { between two plume edges }\end{array}$ & 287.85 & 174.00 & 69.55 \\
\cline { 2 - 5 } & $\begin{array}{c}\text { Central point } \\
\text { between two plume edges }\end{array}$ & 269.02 & 169.36 & 71.53 \\
\hline Without response delay consideration & Minimum response & 331.8 & 161.25 & 63.61 \\
\hline
\end{tabular}

\section{Conclusions}

The strategy plume for tracking in a turbulent environment using an odor sensor with a time constant was developed and evaluated in a simulation framework using computational fluid dynamics. The sensor response delay makes the observed response value unreliable in representing odor concentration dynamics. Therefore, the transient characteristic of sensor response data was investigated to identify a plume. The transient characteristic was observed by calculating the gradient of the data. From this observation, it was found that the gradient sign of a curve-fitted time series response is sufficiently reliable to detect a plume edge. The problem of inaccurate plume identification owing to plume dynamics was addressed by incorporating a redundant check of the gradient sign. This plume detection was used as the input parameter for all switching conditions of the tracking phases and the centerline determination. The evaluation of the tracking strategy in a realistic turbulent environment shows promise with a success rate of around $70 \%$. The success rate is quite high considering the complexity of the environment and that many initial positions of the evaluation are near walls and at the upwind side of the source, which are significant disadvantages for source localization.

The failures were frequent when tracking began at initial positions far from the centerline and at the upwind side of the source. Therefore, further improvement should be made by including a strategy for tracking from these areas. The performance of a plume tracking strategy without response delay consideration was also presented here for comparison. The statistical difference between the performance of the strategy with response delay consideration and without it may not be wide but it is significant and helpful since any actual chemical sensor has response delay. Considering that the statistical result is obtained from a sufficient number of tests covering the entire search area, the proposed delay consideration strategy is likely to consistently provide a clear advantage for different environmental conditions. The difference is expected to be enhanced the faster the sensor node moves. Although actual sensors are not used in our work, the strategy described here is applicable to a sensing system to search for an odor source. 


\section{References}

1 MRIGlobal: Chemical Sensors Market Survey (MRIGlobal, 2013) p. 11.

2 K. S. Eu and K. M. Yap: Int. J. Adv. Rob. Syst. 15 (2018) 1. https://dx.doi.org/10.1177/1729881418755877

3 S. Shigaki, T. Sakurai, N. Ando, D. Kurabayashi, and R. Kanzaki: IEEE Rob. Autom. Lett. 3 (2018) 76. https:// dx.doi.org/10.1109/LRA.2017.2730361

4 H. Ishida, G. Nakayama, and T. Nakamoto: IEEE Sens. J. 5 (2005) 537. https://dx.doi.org/10.1109/ JSEN.2004.839597

5 M. Muhtadi and T. Nakamoto: IEEJ Trans. Sens. Micromachine 138 (2018) 15. https://dx.doi.org/10.1541/ ieejsmas. 138.15

6 M. A. Arain, H. Fan, V. H. Bennetts, E. Schaffernicht, and A. J. Lilienthal: Olfaction and Electronic Nose (ISOEN), ISOCS/IEEE Int. Symp. Olfaction and Electronic Nose (ISOEN) 2017. https://dx.doi.org/10.1109/ ISOEN.2017.7968895

7 Y. Tong, Q. H. Meng, B. Luo, M. Zeng, Y. Wang, and P. F. Qi: Olfaction and Electronic Nose (ISOEN), ISOCS/ IEEE Int. Symp. Olfaction and Electronic Nose (ISOEN) 2017. https://dx.doi.org/10.1109/ISOEN.2017.7968885

8 M. Awadalla, T. F. Lu, Z. Tian, B. Dally, and Z. Liu: Build. Environ. 70 (2013) 10. https://dx.doi.org/10.1016/ j.buildenv.2013.07.021

9 A. Marjovi and L. Marques: European Conf. Mobile Robots (2013) 379. https://dx.doi.org/10.1109/ ECMR.2013.6698871

10 L. Davidson: http://www.tfd.chalmers.se/ lada/postscript_files/solids-and-fluids_turbulent-flow_turbulencemodelling.pdf (accessed March 2018) p. 137.

11 A. Bejan: Convection Heat Transfer (Wiley, New Jersey, 2013) p. 305.

12 Y. A. Cengel and J. M. Cimbala: Fluid Mechanics: Fundamentals and Applications (McGraw-Hill, New York, 2006) p. 324.

13 H. Ishida, T. Nakamoto, and T. Moriizumi: Sens. Actuators, B 49 (1998) 52. https://dx.doi.org/10.1016/S09254005(98)00036-7

\section{About the Authors}

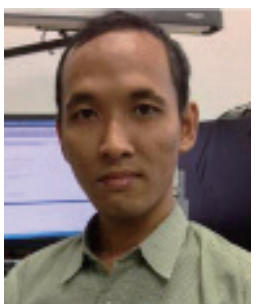

Muis Muhtadi received his B.E. in electrical engineering from Institut Teknologi Sepuluh Nopember Surabaya (ITS), Indonesia, in 2006. In 2010, he entered the master's program in ITS and continued his study at Hochschule Darmstadt (h_da), Germany, in 2011 under a dual degree program from which he received his M.Sc. degree in microelectronics from h_da, and M.E. in telematics from ITS in 2013. He is now pursuing a $\mathrm{PhD}$ degree at Tokyo Institute of Technology. (muis.muhtadi@gmail.com)

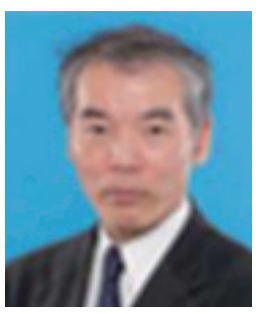

Takamichi Nakamoto received his B.E. and M.E. degrees in 1982 and 1984, respectively, and his Ph.D. degree in electrical and electronic engineering from Tokyo Institute of Technology, Tokyo, Japan. He worked for Hitachi in the area of VLSI design automation from 1984 to 1987. In 1987, he joined Tokyo Institute of Technology as a research associate. In 1993, he became an associate professor with the Department of Electrical and Electronics Engineering, Tokyo Institute of Technology. From 1996 to 1997, he was a Visiting Scientist at Pacific Northwest Laboratories, Richland, WA, USA. He is currently a professor with the Institute for Innovative Research, Tokyo Institute of Technology. (nakamoto@nt.pi.titech.ac.jp) 